\title{
Full thickness skin grafts from the groin: donor site morbidity and graft survival rate from 50 cases
}

\author{
Somi Kim¹, Seung-Won Chung ${ }^{1}$, In-Ho Cha ${ }^{1,2}$ \\ ${ }^{1}$ Department of Oral and Maxillofacial Surgery, ${ }^{2}$ Oral Cancer Research Institute, College of Dentistry, Yonsei University, Seoul, Korea
}

\begin{abstract}
J Korean Assoc Oral Maxillofac Surg 2013;39:21-26)
Objectives: Full thickness skin grafts (FTSG) offer several advantages; they are esthetically superb, have less postoperative shrinkage, and offer minimal postoperative pain and scar formation at the donor site. As a donor site of FTSG, the groin offers a relatively large area of skin with high elasticity. The aim of this study was to evaluate FTSG from the groin for reconstruction in oral and maxillofacial surgery.

Materials and Methods: In a retrospective study, 50 patients (27 males, 23 females) who received FTSG from the groin were evaluated for their operation records, clinical photography, and medical records.

Results: The width of skin from the groin was distributed from 2-8 cm (mean: $5.1 \mathrm{~cm}$ ) at the donor site, while the long axis length was distributed from 3-13 cm (mean: $7.4 \mathrm{~cm}$ ). A high number of patients, 47 patients (94\%) out of 50, showed good healing at the donor site. Wound impairment was seen in 3 patients $(6 \%)$, minor wound dehiscence in 2 patients, and severe wound dehiscence in 1 patient. In the recipient site, delayed healing was observed in 2 patients (4\%).

Conclusion: FTSG from the groin to repair soft tissue defects in reconstruction surgery is a good method due to the relatively big size of the graft, decreasing morbidity at the donor site, and higher graft survival rates.
\end{abstract}

Key words: Skin transplantation, Groin, Forearm, Free tissue flaps, Mouth mucosa

[paper submitted 2012. 4. 17 / revised 2012. 12. 18 / accepted 2013. 1. 7]

\section{Introduction}

Skin grafts are widely used in the field of oral and maxillofacial surgery, from repairing intraoral mucosal defects to coverage of the radial forearm free flap (RFFF) donor sites. Split thickness skin grafts (STSG) generally have higher survival rate, and they are easier to harvest compared to full thickness skin grafts (FTSG). Note, however, that the use of STSG has functional and esthetic complications including esthetically undesirable appearance, severe pain and discomfort in the early postoperative period, delayed wound healing and scar, and pigmentation formation. FTSG can

\section{In-Ho Cha}

Department of Oral and Maxillofacial Surgery, College of Dentistry, Yonsei University, 50 Yonsei-ro, Seodaemun-gu, Seoul 120-752, Korea

TEL: +82-2-2228-3140 FAX: +82-2-2227-7825

E-mail: cha8764@yuhs.ac

(c) This is an open-access article distributed under the terms of the Creative Commons Attribution Non-Commercial License (http://creativecommons.org/licenses/by-nc/3.0/), which permits unrestricted non-commercial use, distribution, and reproduction in any medium, provided the original work is properly cited.

Copyright (C) 2013 The Korean Association of Oral and Maxillofacial Surgeons. All rights reserved. ensure favorable appearance with less shrinkage compared to STSG and less postoperative pain and discomfort of the donor site; on the other hand, the survival rate is lower than STSG, with FTSG having a limitation in terms of skin size; hence the possible difficulty in repairing huge defects. As a donor site of FTSG, the groin offers the advantages of being able to harvest relatively larger amounts of skin compared to other parts of the body, with the scar marked by inguinal crease. Likewise, by using the method suggested by Taifour Suliman $^{1}$, faster healing process could be expected. The authors studied retrospectively 50 cases of FTSG from the groin for reconstruction in the field of oral and maxillofacial surgery and achieved favorable outcome.

\section{Materials and Methods}

From November 2008 to February 2011, 50 patients (27 males, 23 females) who received FTSG from the groin participated in a retrospective study using their operation record, clinical photography, and medical record. Patients'

*This study was supported by a faculty research grant of Yonsei University College of Dentistry for 2008 (6-2008-0061). 

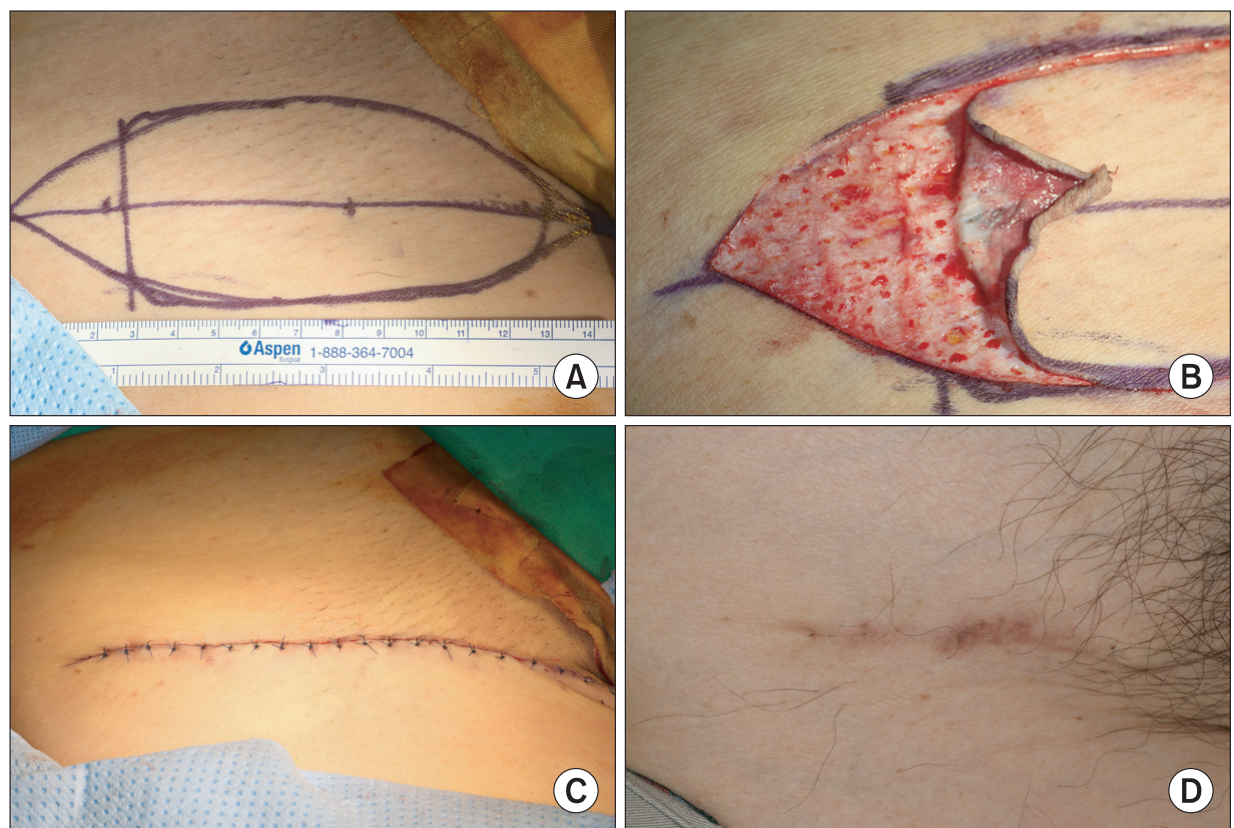

Fig. 1. A. Construction for skin harvesting on groin, using inguinal crease as the long axis. B. Harvesting thin skin without exposing the underlying fat tissue. C. Neighboring and layer-by-layer suturing of wound margins without undermining. D. Healing status of the donor site 1 month after the surgery.

Somi Kim et al: Full thickness skin grafts from the groin: donor site morbidity and graft survival rate from 50 cases. J Korean Assoc Oral Maxillofac Surg 2013 mean age was 61 (youngest: 31 years old; oldest: 87 years old). Surgery was performed under general anesthesia, and we harvested fusiform skin using the inguinal crease of the groin as the long axis.(Fig. 1. A) For skin harvesting, we used the methods suggested by Taifour Suliman ${ }^{1}$ and made an incision with a $45^{\circ}$ angle inside along a boundary of the constructed skin graft and harvested by slicing it. As such, we minimized bleeding and exposure of underlying fat tissues.(Fig. 1. B) After skin harvesting, we controlled hemorrhage using electrocautery; without undermining, the margin areas of the skin consisting of two lines of fusiform were neighbored and sutured layer by layer.(Fig. 1. C) Ten days after surgery, we performed stitch-out of the donor site. We studied the medical record of the donor site as well as the recipient site.

\section{Results}

Table 1 and Table 2 show the distribution of the recipient sites of skin grafts. The width of skin was distributed from $2 \mathrm{~cm}$ to $8 \mathrm{~cm}$ (mean: $5.1 \mathrm{~cm}$ ); the long axis length of the groin was distributed from $3 \mathrm{~cm}$ to $13 \mathrm{~cm}$ (mean: $7.4 \mathrm{~cm}$ ). Among 50 patients, impaired healing of the donor site wound was observed on 3 patients $(6 \%)$. Two patients $(4 \%)$ were found to have minor wound dehiscence, with 1 patient $(2 \%)$ observed to have severe wound impairment that exposed the downward fat tissues because of necrosis on some parts of the skin; they were all healed in 2 months. The remaining 47 patients $(94 \%)$ exhibited favorable healing condition. As to the healing condition of the recipient site, delayed healing was noted on 2 patients (4\%) (skin defect of neck: 1 patient; donor site of RFFF: 1 patient), but both were healed in 2 months.(Table 3)

\section{Discussion}

In the field of oral and maxillofacial surgery, skin grafting is required in the following cases; covering the defect of the vascularized free flap donor site, repairing the exposed raw surface after intraoral mass excision, and covering the defect after the mass excision of the head and neck area or trauma. Whether to use FTSG or STSG is decided based on the size of the skin, available donor site, vascularity of the recipient site, and esthetic considerations. For the coverage of the RFFF donor site, STSG from the thigh is generally used; satisfactory results have been reported regarding the function of the forearm, healing period, and esthetic aspects. The donor site defect of RFFF is preferentially covered by STSG because it can easily be harvested in rectangular form using a dermatome. Moreover, STSG is preferentially used in cases of oral mucosal defects where the mobility of the underlying soft tissue would decrease the survival rate of FTSG. Note, however, that the donor site of STSG has a long healing period and the disadvantages of postoperative pain, scar formation, and esthetic problems ${ }^{2}$. Likewise, STSG requires much more effort in terms of postoperative care of the donor site $^{3}$. FTSG offers the advantages of superb esthetics ${ }^{4}$, less postoperative shrinkage, and minimal postoperative pain and 
Table 1. Study population

\begin{tabular}{|c|c|c|c|c|c|c|}
\hline \multirow{2}{*}{ Age } & \multirow{2}{*}{ Sex } & \multirow{2}{*}{ Type of recipient site } & \multicolumn{2}{|c|}{ Size of harvested skin } & \multirow{2}{*}{$\begin{array}{l}\text { Donor site } \\
\text { healing }\end{array}$} & \multirow{2}{*}{$\begin{array}{l}\text { Recipient site } \\
\text { healing }\end{array}$} \\
\hline & & & Width $(\mathrm{cm})$ & Length $(\mathrm{cm})$ & & \\
\hline 71 & $\mathrm{~F}$ & Donor site of FFF & 3 & 5 & Good & Good \\
\hline 31 & M & Donor site of FFF & 3.5 & 6 & Good & Good \\
\hline 51 & M & Skin defect of neck & 2 & 7 & Severe wound dehiscence & Minor wound dehiscence \\
\hline 66 & M & Oral mucosal defect & 3 & 3 & Good & Good \\
\hline 63 & $\mathrm{~F}$ & Oral mucosal defect & 4 & 8 & Good & Good \\
\hline 68 & $\mathrm{~F}$ & Oral mucosal defect & 4 & 8 & Good & Good \\
\hline 41 & M & Oral mucosal defect & 3 & 5 & Good & Good \\
\hline 68 & M & Oral mucosal defect & 5 & 6 & Good & Good \\
\hline 74 & M & Oral mucosal defect & 5 & 7 & Good & Good \\
\hline 60 & $\mathrm{~F}$ & Oral mucosal defect & 6 & 8 & Good & Good \\
\hline 41 & $\mathrm{~F}$ & Oral mucosal defect & 3 & 4 & Minor wound dehiscence & Good \\
\hline 33 & $\mathrm{~F}$ & Oral mucosal defect & 3 & 6 & Minor wound dehiscence & Good \\
\hline 75 & $\mathrm{~F}$ & Oral mucosal defect & 5 & 7 & Good & Good \\
\hline 81 & M & Oral mucosal defect & 4 & 6 & Good & Good \\
\hline 54 & $\mathrm{~F}$ & Oral mucosal defect & 4 & 7 & Good & Good \\
\hline 81 & $\mathrm{~F}$ & Oral mucosal defect & 5 & 8 & Good & Good \\
\hline 53 & $\mathrm{~F}$ & Oral mucosal defect & 4 & 7 & Good & Good \\
\hline 56 & M & Oral mucosal defect & 2.5 & 4 & Good & Good \\
\hline 48 & M & Oral mucosal defect & 4 & 8 & Good & Good \\
\hline 75 & M & Oral mucosal defect & 4 & 5 & Good & Good \\
\hline 50 & M & Oral mucosal defect & 6 & 10 & Good & Good \\
\hline 59 & $\mathrm{~F}$ & Oral mucosal defect & 6 & 13 & Good & Good \\
\hline 71 & $\mathrm{~F}$ & Oral mucosal defect & 4 & 10 & Good & Good \\
\hline 72 & $\mathrm{~F}$ & Oral mucosal defect & 3 & 4 & Good & Good \\
\hline 38 & M & Donor site of RFFF & 4 & 5 & Good & Good \\
\hline 73 & $\mathrm{~F}$ & Donor site of RFFF & 3 & 4 & Good & Minor wound dehiscence \\
\hline 57 & $\mathrm{~F}$ & Donor site of RFFF & 6 & 9 & Good & Good \\
\hline 73 & $\mathrm{~F}$ & Donor site of RFFF & 6 & 9 & Good & Good \\
\hline 73 & $\mathrm{~F}$ & Donor site of RFFF & 6 & 7.5 & Good & Good \\
\hline 59 & M & Donor site of RFFF & 6 & 8 & Good & Good \\
\hline 49 & M & Donor site of RFFF & 5 & 6 & Good & Good \\
\hline 69 & $\mathrm{~F}$ & Donor site of RFFF & 6 & 8 & Good & Good \\
\hline 59 & M & Donor site of RFFF & 6 & 8 & Good & Good \\
\hline 57 & M & Donor site of RFFF & 7 & 9 & Good & Good \\
\hline 66 & M & Donor site of RFFF & 6.5 & 9 & Good & Good \\
\hline 68 & M & Donor site of RFFF & 6.5 & 8.5 & Good & Good \\
\hline 83 & $\mathrm{~F}$ & Donor site of RFFF & 7 & 10 & Good & Good \\
\hline 64 & $\mathrm{~F}$ & Donor site of RFFF & 8 & 10 & Good & Good \\
\hline 58 & M & Donor site of RFFF & 8 & 10 & Good & Good \\
\hline 60 & $\mathrm{~F}$ & Donor site of RFFF & 4 & 5 & Good & Good \\
\hline 73 & M & Donor site of RFFF & 5 & 8 & Good & Good \\
\hline 62 & M & Donor site of RFFF & 4 & 6 & Good & Good \\
\hline 71 & M & Donor site of RFFF & 5 & 6 & Good & Good \\
\hline 87 & M & Donor site of RFFF & 7 & 8 & Good & Good \\
\hline 56 & M & Donor site of RFFF & 6 & 8 & Good & Good \\
\hline 64 & M & Donor site of RFFF & 6 & 6 & Good & Good \\
\hline 41 & M & Donor site of RFFF & 7 & 9 & Good & Good \\
\hline 67 & $\mathrm{~F}$ & Donor site of RFFF & 7 & 9 & Good & Good \\
\hline 52 & M & Donor site of RFFF & 8 & 10 & Good & Good \\
\hline 48 & $\mathrm{~F}$ & Donor site of RFFF & 8 & 10 & Good & Good \\
\hline
\end{tabular}

(F: female, M: male, FFF: fibula free flap, RFFF: radial forearm free flap)

Somi Kim et al: Full thickness skin grafts from the groin: donor site morbidity and graft survival rate from 50 cases. J Korean Assoc Oral Maxillofac Surg 2013

scar formation through primary closure compared to $\mathrm{STSG}^{5,6}$. Nonetheless, the size of FTSG is relatively limited, and there is a possibility of graft failure due to the thickness of the graft ${ }^{7}$.

The groin offers relatively larger size of skin due to surplus skin with high elasticity. After skin harvesting, the defect could primarily be closed, and the scar could be hidden by the natural crease ${ }^{8}$. Kim et al. ${ }^{6}$ performed FTSG from the groin for coverage of RFFF in 40 patients and reported no postoperative pain or limitation of movement on the groin 
and 2 cases of tendon exposure on the forearm. The authors implemented FTSG but chose the groin as donor site, which has extra skin; thus gaining relatively big graft and using it on the RFFF donor site or oral mucosal defect. Moreover, they minimized the thickness of the graft using Suliman's method to increase the survival rate of graft. Taifour Suliman ${ }^{1}$ introduced a simple method of harvesting skin by making an incision with a $45^{\circ}$ angle and detaching the skin by slicing it to gain relatively thin skin, which would reduce fat exposure with no bleeding.

At least 47 patients (94\%) out of 50 exhibited good healing process of the donor site.(Fig. 1. D) Wound impairment was

Table 2. Summary of the study population

\begin{tabular}{lc}
\hline Age (mean age) (yrs) & $31-87$ (61) \\
Sex (n) & 27 \\
Males & 23 \\
Females & \\
Type of recipient site (n) & 27 \\
Donor site of RFFF & 20 \\
Oral mucosal defect & 2 \\
Donor site of FFF & 1 \\
Skin defect of neck & \\
Size of harvested skin (cm) & $2.5-8.0(5.1)$ \\
Width (mean) & $3.0-13.0(7.4)$ \\
Length (mean) & \\
\hline (RFFF: radial forearm free flap, FFF: forearm free flap) \\
Somi Kim et al: Full thickness skin grafts from the groin: donor site morbidity and graft \\
survival rate from 50 cases. J Korean Assoc Oral Maxillofac Surg 2013
\end{tabular}

Table 3. Result summary

\begin{tabular}{lc}
\hline & $\mathrm{n}(\%)$ \\
\hline Donor site healing & $50(100)$ \\
Good & $47(94)$ \\
Minor wound & $2(4)$ \\
Severe wound & $1(2)$ \\
Recipient site healing & $50(100)$ \\
Good & $48(96)$ \\
Minor wound & $2(4)$ \\
Severe wound & $0(0)$ \\
\hline
\end{tabular}

Somi Kim et al: Full thickness skin grafts from the groin: donor site morbidity and graft survival rate from 50 cases. J Korean Assoc Oral Maxillofac Surg 2013. J Korean Assoc Oral Maxillofac Surg 2013 noted on 3 patients (6\%), minor wound dehiscence, on 2 patients, and severe wound dehiscence, on 1 patient. Minor wound dehiscence ( 2 cases) seemed to have no correlation with the size of the graft since the latter was relatively small (width of $3.0 \mathrm{~cm}$, length of $4.0 \mathrm{~cm}$; width of $3.0 \mathrm{~cm}$, length of $6.0 \mathrm{~cm}$ each). On the other hand, because the patients were young (one is a 33-year-old woman, the other a 41-yearold woman), we think there was a lot of tension between two margins of skin. One case of severe wound dehiscence (51-year-old man) was observed on the graft measuring $2.0 \times 7.0 \mathrm{~cm}$, with partial necrosis of the skin margin and exposed underneath fat tissue noted. In this case, the width of harvested skin was relatively small. Thus, we think the size of skin was not the cause of wound dehiscence. On the other hand, the patient has many other wound dehiscences on his body such as neck wound (recipient site of skin graft), chest wound formed due to the raising of pectoralis major myocutaneous flap, and intraoral wound that developed due to tumor resection and reconstruction. As such, we concluded that, even though he has no systemic disease, he has a systemic problem with the wound healing process.

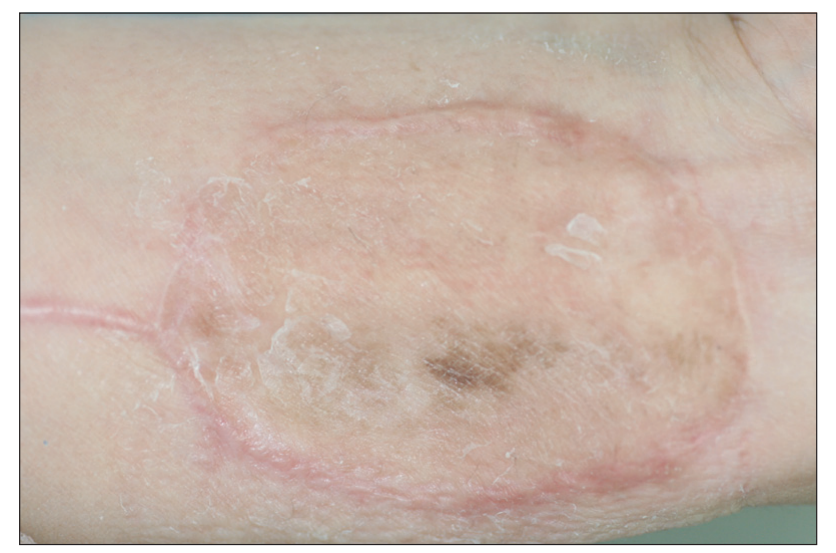

Fig. 3. Healing status of the recipient site (donor site of radial forearm free flap) 4 months after the surgery.

Somi Kim et al: Full thickness skin grafts from the groin: donor site morbidity and graft survival rate from 50 cases. J Korean Assoc Oral Maxillofac Surg 2013
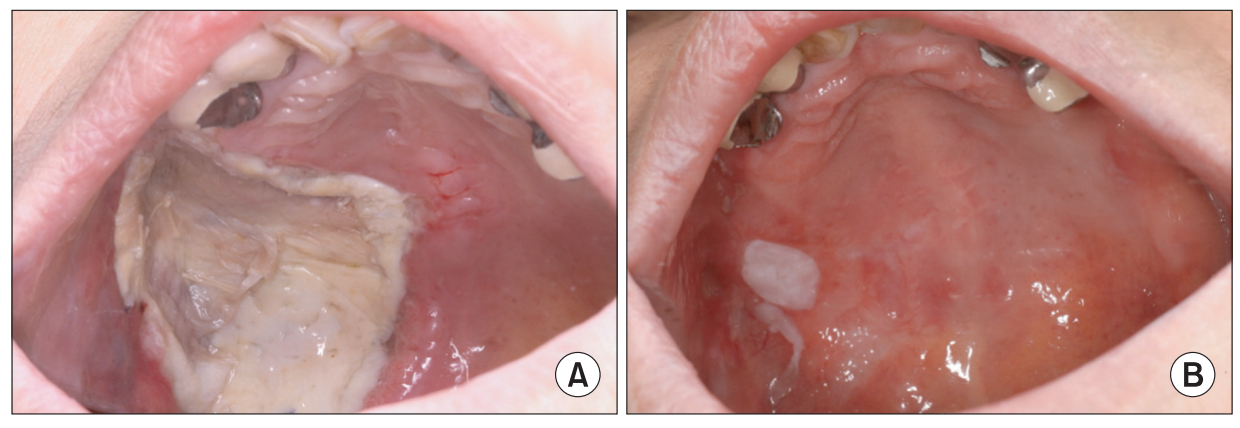

Fig. 2. A. Healing status of the recipient site (oral mucosal defect) 10 days after the surgery. B. Healing status of the recipient site (oral mucosal defect) 2 months after the surgery.

Somi Kim et al: Full thickness skin grafts from the groin: donor site morbidity and graft survival rate from 50 cases. J Korean Assoc Oral Maxillofac Surg 2013 
A total of 48 patients $(96 \%)$ succeeded in grafting, with good healing process of the recipient site (Figs. 2, 3), with 2 patients (4\%) exhibiting graft failure and impaired wound healing. One patient showed partial exposure of the tendon on the RFFF defect site, whereas the other manifested grafting failure on skin defect of neck; the latter was the same patient who showed severe wound impairment of the groin. For this patient, the general condition probably influenced wound healing.

For wound closure, we pulled both sides of the wound edges without undermining the tissue underneath and performed primary closure. The reason for not carrying out undermining - which reduces tension of tissue - is that, since groin has surplus skin, wound margins can be easily brought together without undermining. In addition, the textbook meaning of undermining suggests the implementation of wound closure by doing dissection on the skin and fat layer in the plane of the upper muscle under no tension. Undermining involves dissecting the skin and fat layer to reduce tension during wound closure. In the groin, however, undermining may damage the superficial epigastric vessels and superficial circumflex iliac vessels lying above the inguinal ligament. Moreover, this takes much time and has the weakness of causing profuse bleeding because of the thick fat layer, which may cause excessive bleeding during undermining. In the clinic, we attempted wound closure after undermining the fat layer right underneath the skin but encountered skin necrosis due to the lack of blood supply on the skin margins. Therefore, we performed wound closure without undermining for the 50 cases. Inguinal crease had harvested mean width of $5.1 \mathrm{~cm}$ and actually yielded good results in the biggest width of $8.0 \mathrm{~cm}$. The harvested skin was measured to have mean width of $5.1 \mathrm{~cm}$ and maximum width of $8.0 \mathrm{~cm}$.

Taifour Suliman's method of skin harvesting is controversial. In this method, deep dermis is not entirely included in the graft, possibly leaving a dermal layer on the donor site; this in turn can lead to the formation of epidermal inclusion cyst. Still, Taifour Suliman ${ }^{1}$ insisted that the layer left after skin harvest is part of superficial fascia, not deep dermis; hence the extremely low possibility of inclusion cyst formation, Taifour Suliman also mentioned that none of the above occurred in the reported cases. In our 50 cases, the occurrence of epidermal inclusion cyst was not observed.

There are limitations with FTSG as well. Since the groin is hair-bearing tissue, undesirable hair growth may occur on the recipient sites. Furthermore, skin from the groin has a tendency of dark pigmentation ${ }^{8}$. The inner surface of the upper arm may be considered an alternative donor site with esthetically good skin, but harvesting a large size of skin is difficult. The lower abdomen is another alternative; although large skin harvest is possible, however, it does not match the skin of the head and neck region. To do away with the need for an additional donor site for grafting, primary closure may be considered in small radial defects ${ }^{9}$. In medium-sized radial defects, Ular flap ${ }^{10}$, Z-plasty ${ }^{11}$, or modified Z-plasty of combined Ular flap and Z-plasty ${ }^{12}$ were introduced. Note, however, that local flap requires wide dissection underneath the local flap. In larger radial defects, grafting full thickness skin from the proximal side of the defect area was introduced by using the concept of autonomous reparative unit, which allows accurate and efficient design of the graft ${ }^{13}$. Split thickness skin of the radial flap may also be harvested prior to radial flap raising ${ }^{14}$. Split thickness skin was harvested in mesh form from 66 patients, with its size stretched or grafted with non-mesh form. Because of the uneven surface of the forearm, they posed a difficulty to site dermatome; hence the failed harvesting skin graft from one old man.

\section{Conclusion}

We think FTSG from the groin for soft tissue defect repair in reconstruction surgery is a good method considering the relatively big graft obtained, lower morbidity of the donor site, and higher graft survival rate. Nonetheless, we think the size of the skin graft should not be big when applied to younger patients since wound dehiscences could develop. After surgery, all patients had no problem walking, but they were not able to wear triangle briefs since such may lead to wound impairment. We believe our results using FTSG from the groin for reconstructive surgery demonstrate that this is a favorable technique for both donor and recipient sites.

\section{References}

1. Taifour Suliman M. A simple method to facilitate full-thickness skin graft harvest. Burns 2009;35:87-8.

2. Avery CM. Review of the radial free flap: is it still evolving, or is it facing extinction? Part one: soft-tissue radial flap. Br J Oral Maxillofac Surg 2010;48:245-52.

3. Sidebottom AJ, Stevens L, Moore M, Magennis P, Devine JC, Brown JS, et al. Repair of the radial free flap donor site with full or partial thickness skin grafts. A prospective randomised controlled trial. Int J Oral Maxillofac Surg 2000;29:194-7.

4. Lutz BS, Wei FC, Chang SC, Yang KH, Chen IH. Donor site morbidity after suprafascial elevation of the radial forearm flap: a prospective study in 95 consecutive cases. Plast Reconstr Surg 1999;103:132-7.

5. Avery CM, Iqbal M, Orr R, Hayter JP. Repair of radial free flap 
donor site by full-thickness skin graft from inner arm. Br J Oral Maxillofac Surg 2005;43:161-5.

6. Kim TB, Moe KS, Eisele DW, Orloff LA, Wang SJ. Full-thickness skin graft from the groin for coverage of the radial forearm free flap donor site. Am J Otolaryngol 2007;28:325-9.

7. Ratner D. Skin grafting. Semin Cutan Med Surg 2003;22:295-305.

8. Palkar VM. Full-thickness skin grafting. J Surg Oncol 2000;73:31.

9. Shiba K, Iida Y, Numata T. Ipsilateral full-thickness forearm skin graft for covering the radial forearm flap donor site. Laryngoscope 2003;113:1043-6.

10. Juretic M, Car M, Zambelli M. The radial forearm free flap: our experience in solving donor site problems. J Craniomaxillofac Surg 1992;20:184-6.

11. Hui KC, Zhang F, Lineaweaver WC. Z-plasty closure of the donor defect of the radial forearm free flap. J Reconstr Microsurg 1999;15:19-21.

12. Bashir MA, Fung V, Kernohan MD, Ragbir M, Ahmed OA "Z-plasty" modification of ulnar-based fasciocutaneous flap for closure of the radial forearm flap donor defect. Ann Plast Surg 2010;64:22-3.

13. Squadrelli-Saraceno M, Compan A, Bimbi G, Gatto L, Riccio S, Colombo S. Autonomous reparative unit (ARU): a new concept of repairing free flap donor site with local full-thickness skin graft. Acta Otorhinolaryngol Ital 2010;30:40-6.

14. Ghanem TA, Wax MK. A novel split-thickness skin graft donor site: the radial skin paddle. Otolaryngol Head Neck Surg 2009;141:390-4. 